\title{
Proximity effect in ion-beam-induced deposition of nanopillars
}

\author{
Ping Chen, ${ }^{a}$ Huub W. M. Salemink, and Paul F. A. Alkemade \\ Kavli Institute of Nanoscience, Delft University of Technology, Lorentzweg 1, 2628 CJ Delft, \\ The Netherlands
}

(Received 12 January 2009; accepted 26 May 2009; published 24 June 2009)

\begin{abstract}
Ion-beam-induced deposition (IBID) is a powerful technique for prototyping three-dimensional nanostructures. To study its capability for this purpose, the authors investigate the proximity effect in IBID of nanopillars. In particular, the changes in shape and dimension of pillars are studied when a second pillar is grown near an existing pillar. On a semiconducting bulk $\mathrm{Si}$ and on an insulating $\mathrm{Si}_{3} \mathrm{~N}_{4}$ membrane the first pillar gets broader, whereas on $\mathrm{Si}$ it starts to bend. They attribute the broadening and bending to the additional deposition induced by the particles scattered from the growing second pillar. On $\mathrm{Si}$ the second pillar is taller than the first one, while on $\mathrm{Si}_{3} \mathrm{~N}_{4}$ it is shorter and rougher. This difference points to an important role of the substrate conductivity in the proximity effect. In a conductive environment the changes in the second pillar are mainly caused by a precursor coverage enhancement in the pillar surface. This enhancement is caused by precursor molecules, which are reflected or desorbed from the first pillar. In the case of an insulating environment, the changes in the second pillar are mainly caused by the reduction in the substrate surface charging due to the presence of the first pillar. () 2009 American Vacuum Society.
\end{abstract}

[DOI: $10.1116 / 1.3155825]$

\section{INTRODUCTION}

The direct writing technology ion-beam-induced deposition (IBID) was first demonstrated by Gamo et al. ${ }^{1}$ Precursor molecules absorbed on a substrate surface are decomposed by an ion-beam-induced reaction, resulting in localized material deposition. The most important characteristic of IBID is its high flexibility for the shape and location of the deposited structures. Hence, IBID gains much interest as a powerful tool for prototyping three-dimensional (3D) nanostructures for various applications. ${ }^{2,3}$ Examples are a nanoelectrostatic actuator and a nanospace wiring as parts of a nanomechanical system, ${ }^{4}$ a cantilever as a mass sensor, ${ }^{5}$ a four-wing rotor, ${ }^{6}$ an electrostatically actuated nanomanipulator, ${ }^{7}$ bionanotools as a nanoinjector, ${ }^{8}$ a nanonet, ${ }^{9}$ a nanofilter, ${ }^{10}$ a nanosensing probe,${ }^{11}$ and a field emitter. ${ }^{12,13}$

A pillar can be regarded as a simple example of 3D nanostructures. However, IBID pillars are often not as regular as intended. For instance, they frequently exhibit irregular extensions on their sidewalls and micrometer-sized halos, namely, deposits around their bases. We previously reported that smooth and narrow pillars with smaller halos can be fabricated under charging conditions on insulating substrates. ${ }^{14}$ We frequently observed changes in shape and dimension when a second pillar grows near an existing pillar. Despite the fundamental drive to improve the quality of complex IBID 3D nanostructures, the knowledge on this proximity effect in IBID pillar growth is still lacking. So far, the proximity effect has only been reported for electron-beaminduced deposition (EBID) pillar growth ${ }^{15-17}$ but not for IBID. We note that these effects are commonly encountered in IBID repair of photolithography masks and integrated circuit modification. Kislov and Khodos ${ }^{15}$ observed that an ex-

${ }^{\text {a) Electronic mail: p.chen@tudelft.nl }}$ isting tungsten pillar gets broader and bends toward a neighboring new pillar. The same effect was observed for carbon pillars by Mitsuishi et al. ${ }^{16}$ Both authors considered the broadening as the result of additional deposition induced by secondary electrons emitted from the new growing pillar. ${ }^{15,16}$ They attributed the bending to an electrostatic force between the two neighboring pillars. ${ }^{15,16}$ Lau et al. ${ }^{17}$ observed that an existing cobalt-containing pillar was broadened and had a lower metal content as compared to the nearby newly grown pillar. They explained this effect as the result of additional deposition on the first pillar during the growth of the second pillar. $^{17}$

In this article we report our investigation on the proximity effect of IBID pillar growth. This study is necessary in order to explore the capabilities and limitations of IBID to fabricating complex 3D nanostructures. Besides the changes in shape and dimension of the existing pillar, we observed also changes in the newly grown pillar. We have compared growth of pillar pairs on substrates with different conductivities. Effects of factors, such as scattering of particles (primary ions, secondary electrons, and atoms), precursor coverage, and substrate charging, are discussed.

\section{EXPERIMENT}

The experiments were performed in a scanning electron microscope (SEM) with a focused-ion-beam (FIB) system (STRATA DualBeam DB235 from FEI). The metal-organic precursor gas $\left(\mathrm{CH}_{3}\right)_{3} \mathrm{Pt}\left(\mathrm{CpCH}_{3}\right)$ is used as gas source. Pillars were grown using a $1 \mathrm{pA} 30 \mathrm{keV} \mathrm{Ga}^{+} \mathrm{FIB}$ at normal incidence in spot mode. The estimated beam diameter was 10 $\mathrm{nm}$. The substrates used in this work were a semiconducting $\mathrm{Si}$ wafer with or without a $30 \mathrm{~nm} \mathrm{Cu}$ coating layer and a $\mathrm{Si}$ wafer with a 45-nm-thick $\mathrm{Si}_{3} \mathrm{~N}_{4}$ top layer and a 400 $\times 400 \mu \mathrm{m}^{2} \mathrm{Si}_{3} \mathrm{~N}_{4}$ membrane window. 


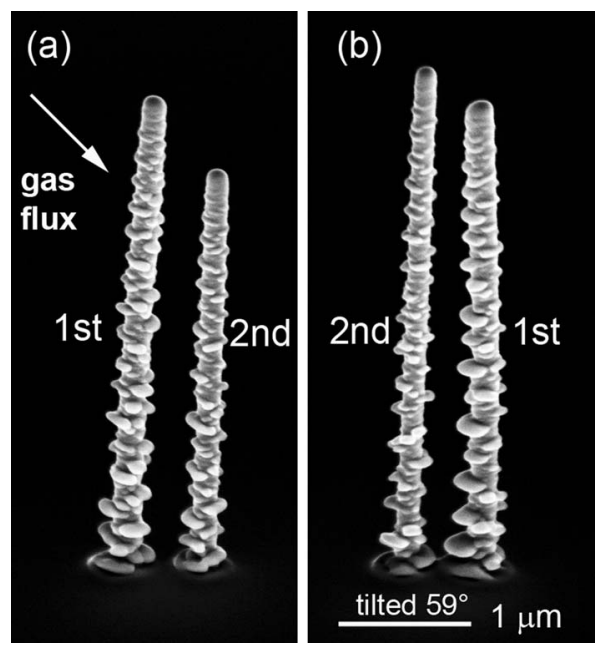

FIG. 1. Shadowing effect in IBID pillar growth. SEM images of two pairs of pillars that are grown in different orders (nozzle is located at the left side) on $\mathrm{Si}$; the second pillar is grown (a) at the left side of the first pillar and (b) at the right side of the first pillar. (Viewing angle is $59^{\circ}$ with respect to the pillar direction.)

To vary the surface charging conditions on the insulating $\mathrm{Si}_{3} \mathrm{~N}_{4}$ surface, some pillars were grown near a conductive $\mathrm{Pt}$ line, which connects the $\mathrm{Si}_{3} \mathrm{~N}_{4}$ window to the bulk $\mathrm{Si}$ surface. In some other cases, a similar Pt line was laid down near the pillars after their growth to enhance their visibility during SEM imaging. The chamber pressure was 3.3 $\times 10^{-6}$ mbar during growth; the background pressure was $5.5 \times 10^{-7}$ mbar. To ensure a constant precursor gas supply, the gas was introduced into the chamber $60 \mathrm{~s}$ before the ion beam. The outlet of a nozzle of about $500 \mu \mathrm{m}$ in diameter was located $430 \mu \mathrm{m}$ above the sample surface, at the left side in all images taken. All pillars were grown in spot mode for $180 \mathrm{~s}$ at room temperature on the same sample. The pillar separation was selected by moving the sample stage instead of shifting the ion beam. Thus, the distance between the nozzle and the beam impact point was the same for every pillar. Tilted $59^{\circ} \mathrm{SEM}$ images were taken for determining the diameter and height of the grown pillars. The measurement of the pillar diameter did not include the sidewall extensions [see Fig. 2(a)].

\section{RESULTS}

To investigate the influence of the direction of the precursor gas flow, we compared two pairs of pillars grown on a bulk Si wafer. One pair was grown from left to right, the other from right to left [see Figs. 1(a) and 1(b)]. The second pillar in Fig. 1(a) is shorter. Likely, it was shadowed from the precursor gas flow by the existing pillar. To avoid this known shadowing effect, ${ }^{18}$ the order of growth is always from right to left in the images in Figs. 2-4.

Figure 2(a) shows pairs of pillars that were grown on $\mathrm{Si}$ at different separations. A single pillar is given for comparison. As a consequence of the growth of the second pillar, the first pillar gets broader and rougher and bends slightly toward the second one for the separation of less than $1.4 \mu \mathrm{m}$. More-
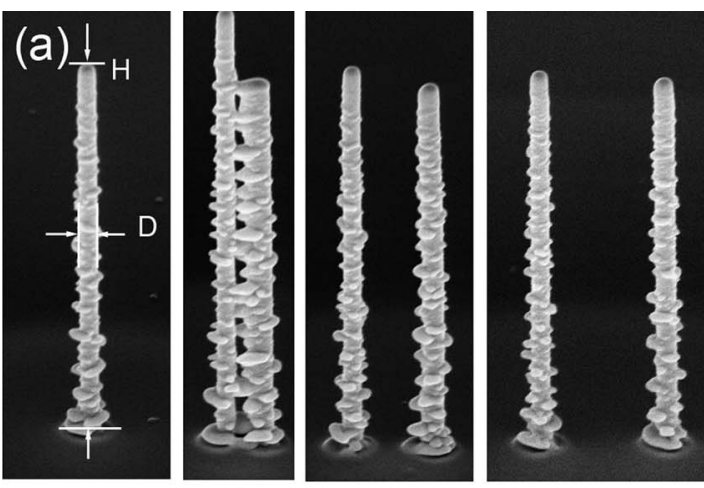

$0.3 \mu \mathrm{m} \quad 0.7 \mu \mathrm{m}$

$1.0 \mu \mathrm{m}$

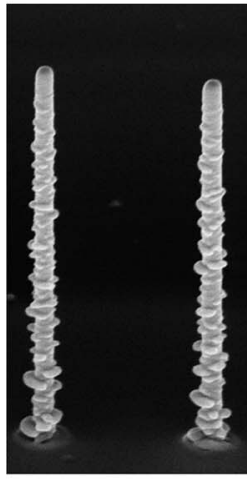

$1.4 \mu \mathrm{m}$

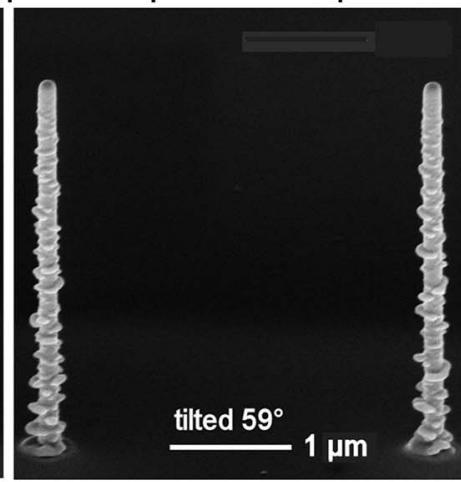

$3.0 \mu \mathrm{m}$ (b)

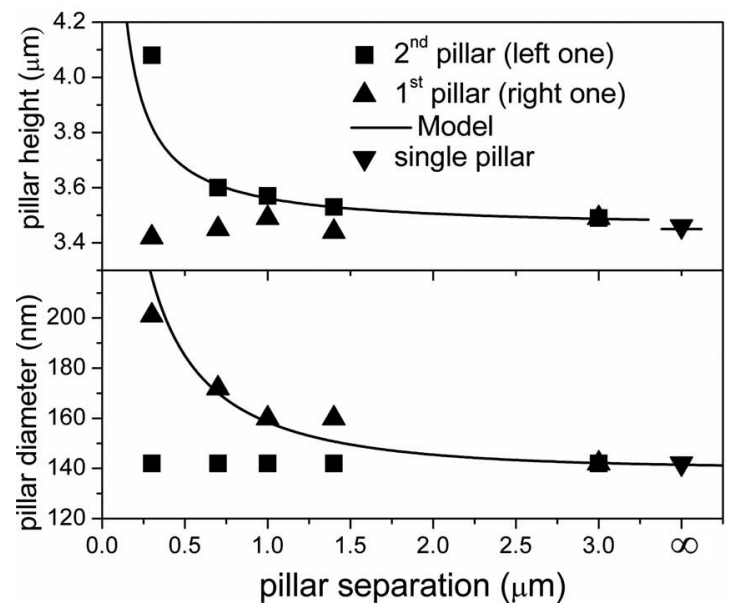

FIG. 2. Proximity effect of pillars grown on a semiconducting bulk Si. (a) SEM images of a single pillar and of five pairs of pillars with different separations from 0.3 to $3 \mu \mathrm{m}$. (b) Relationship between diameter $(D)$ and height $(H)$ of the pillars and pillar separation; the curves are model calculations discussed in the text.

over, the second pillar is taller than the first one. Not surprisingly, the additional length varies with the separation [see Fig. 2(b)]. However, the height of the first pillar and the width of the second pillar are unaffected as compared to those of a single pillar. No proximity effect has been observed when pillars are $3.0 \mu \mathrm{m}$ apart. The halo regions of the pillars are visible in the corresponding top views [see Fig. 2(c)]. Note that if the halo of the second pillar does not extend to the irradiation point of the first pillar, there is no proximity effect visible. 


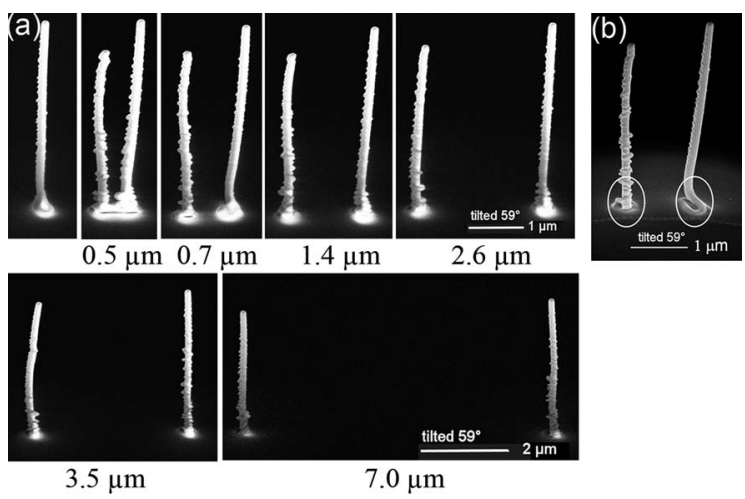

FIG. 3. Proximity effect of pillars grown on a 45 -nm-thick insulating $\mathrm{Si}_{3} \mathrm{~N}_{4}$ membrane. SEM images of (a) six pairs of pillars with different separations from 0.5 up to $7.0 \mu \mathrm{m}$. (b) Different pillar bases.

Figure 3(a) show pairs of pillars that were grown on the insulating $\mathrm{Si}_{3} \mathrm{~N}_{4}$ membrane. In contrast to the pillars on $\mathrm{Si}$, the first pillar broadens only slightly and does not bend toward, but away from the second pillar. Surprisingly, the second pillar is shorter and rougher. Further, its shape is twisted; first, it bends away from the first pillar and then back. In addition, the bases of the neighboring pillars are very different [see in Fig. 3(b)]. Finally, this proximity effect extends up to $7.0 \mu \mathrm{m}$. Even when the halo regions of two neighboring pillars do not overlap, the proximity effect is still pronounced.

The proximity effect on a Si wafer coated with $30 \mathrm{~nm} \mathrm{Cu}$ is very similar to that on a bare $\mathrm{Si}$ wafer [see Fig. 4(a)]. Contrary to the observation of Mitsuishi et al. ${ }^{16}$ for EBID pillars, the bending of the first pillar remains unaffected with increasing substrate conductivity. On the same $\mathrm{Si}_{3} \mathrm{~N}_{4}$ membrane in Fig. 3, two pillars were deposited $7.0 \mu \mathrm{m}$ apart near a Pt line, which connects the $\mathrm{Si}_{3} \mathrm{~N}_{4}$ window to the bulk $\mathrm{Si}$ surface. In contrast to the pillars grown on the bare $\mathrm{Si}_{3} \mathrm{~N}_{4}$ surface, no proximity effect is visible [see Fig. 4(b)]. Figure 4(c) compares a pillar on $\mathrm{Si}$, a pillar pair on $\mathrm{Si}$, a pair on bare $\mathrm{Si}_{3} \mathrm{~N}_{4}$, and a single pillar on $\mathrm{Si}_{3} \mathrm{~N}_{4}$ with a Pt line. The heights of the pillars are similar, apart from the second pillar on $\mathrm{Si}$ and the first pillar on bare $\mathrm{Si}_{3} \mathrm{~N}_{4}$.

\section{DISCUSSION}

IBID results from the interaction of ions with precursor gas molecules absorbed on a substrate surface. The shape and dimension of IBID structures are determined by (a) scattering of primary and secondary particles, (b) precursor coverage, and (c) substrate surface conditions. We discuss the proximity effect in IBID pillar growth in terms of these factors.

\section{A. Effect of scattered particles}

Possible operative mechanisms in IBID are decompositions of adsorbed molecules by (1) primary ions, (2) sputtered atoms or ions, and (3) secondary electrons. ${ }^{19}$ One or

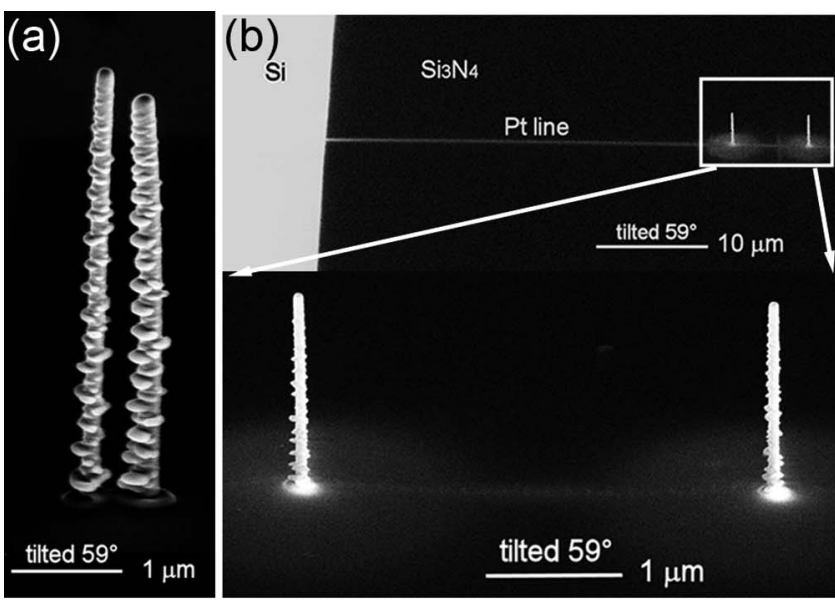

$\mathrm{Si}$ with $\mathrm{Cu}$

$\mathrm{Si}_{3} \mathrm{~N}_{4}$ with a Pt line

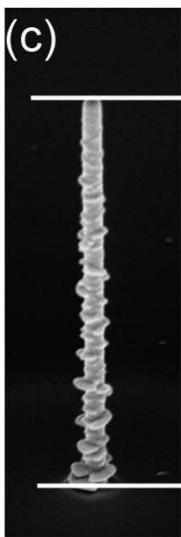

$\mathrm{Si}$

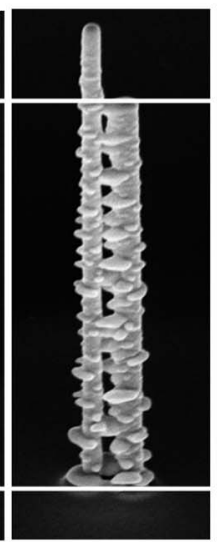

$\mathrm{Si}$

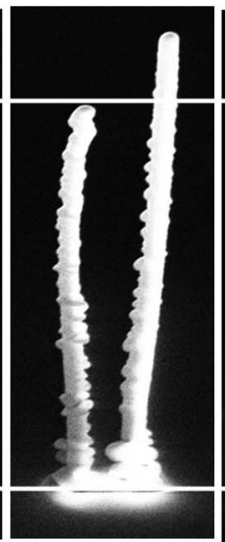

$\mathrm{Si}_{3} \mathrm{~N}_{4}$

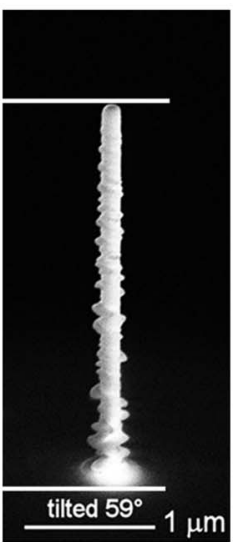

$\mathrm{Si}_{3} \mathrm{~N}_{4}$ with a Pt line
FIG. 4. Role of substrate conductivity in the proximity effect. SEM images of (a) one pair of pillars grown on $\mathrm{Cu}$ coated $\mathrm{Si}$, (b) one pair of pillars grown on $\mathrm{Si}_{3} \mathrm{~N}_{4}$ membrane with a $\mathrm{Pt}$ line, and (c) pillars grown on $\mathrm{Si}$ without or with a neighboring pillar and on $\mathrm{Si}_{3} \mathrm{~N}_{4}$ membrane with a neighboring pillar or a Pt line.

more types of particles can contribute to IBID. In previous work, we have observed significant deposition outside the irradiated area during IBID. ${ }^{20}$

During the growth of the second pillar, some scattered primary ions and emitted secondary electrons and atoms can arrive at the neighboring previously grown pillar. These particles induce additional deposition on the first pillar, resulting in a broadening [see Figs. 2(a) and 3(a)]. A similar broadening of the first pillar was observed in EBID pillar growth. ${ }^{15-17}$ For a smaller pillar separation, a larger fraction of the scattered particles can arrive at the first pillar. Therefore, the broadening scales roughly inversely with the separation [see Fig. 2(a)].

The angular distribution of the emitted secondary particles has an overcosine shape,

$$
\frac{n+1}{2 \pi} \cos ^{n} \theta
$$

where $n=1$ for secondary electrons $\mathrm{s}^{21,22}$ and $1<n<2$ for sputtered atoms. ${ }^{23}$ From this distribution plus geometric fac- 


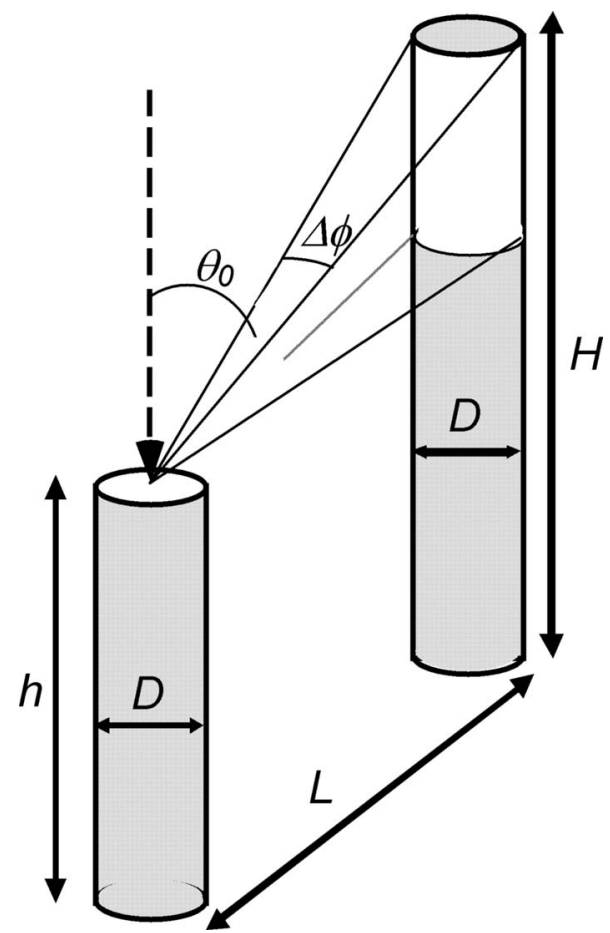

FIG. 5. Proximity effect. Secondary particles are emitted from the growing second pillar (left). If the polar emission angle is between $\theta_{0}$ and $\pi / 2$ and if the azimuthal angle is within the shown range of $\Delta \phi(=D / L)$, the emitted particles arrive at the first pillar (right), causing additional growth in the white colored region.

tors, one can write the fraction of the secondary particles $f_{s}$ emitted from the top of the growing pillar that arrives at the first pillar (see Fig. 5). It is

$$
f_{s}=\int_{-D / 2 L}^{D / 2 L} \int_{\theta_{0}}^{\pi / 2} \frac{n+1}{2 \pi} \cos ^{n} \theta \sin \theta d \theta d \varphi=\frac{D}{2 \pi L} \cos ^{n+1} \theta_{0},
$$

where

$$
\theta_{0}=\arctan \left(\frac{L}{H-h}\right)
$$

The top of the pillar is dome shaped. Because the diameter of the emitting area is much smaller than the pillar diameter, convolution of Eq. (1) with the actual orientations of the emitting surface has only a marginal effect and, thus, can be discarded. Hence, the number $\Delta N_{s}$ of secondary particles that arrive at the first pillar in a time interval $\Delta t$ is

$$
\Delta N_{s}=Y \frac{i_{p} \Delta t}{e} \frac{D}{2 \pi L} \cos ^{n+1} \theta_{0}
$$

where $Y$ is the total number of emitted secondary particles per incident ion, $i_{p}$ is the primary ion current, and $e$ is the elemental charge. We will denote by $\alpha$ the volume growth of the first pillar when it is hit by a secondary particle. The additional volume gain $V_{s}$ of the first pillar during growth of the second pillar is then

$$
\begin{aligned}
V_{s} & =\alpha Y \frac{i_{p}}{e} \frac{D}{2 \pi L} \int \cos ^{n+1} \theta_{0} d t \\
& =\alpha Y \frac{i_{p}}{e} \frac{D}{2 \pi L} \frac{1}{v} \int_{0}^{H} \cos ^{n+1} \theta_{0} d h,
\end{aligned}
$$

where $v$ is the growth speed of the pillar height. The growth speed of the volume of cylindrical pillar is $\frac{1}{4} \pi D^{2} v$. If we denote the volume change in the second pillar per incident ion by $\beta$, we have $i_{p} / e v=\pi D^{2} / 4 \beta$. Hence, Eq. (3) can be written as

$$
V_{s}=\frac{\alpha Y}{\beta} \frac{\pi D^{2}}{4} \frac{D}{2 \pi} \frac{1}{L} \int_{0}^{H} \cos ^{n+1} \theta_{0} d h \equiv \frac{\alpha Y}{\beta} \frac{D^{3}}{8} X(L / H) .
$$

The dimensionless function $X$ depends only on the ratio $L / H$ and can be numerically evaluated. The curve in Fig. 2(b) (top) shows the calculated width of the first pillar after growth of the second pillar according to Eq. (4) with $n$ $=1.5$. We have used $\alpha Y / \beta$ as a fitting parameter. The agreement between the experimental and the calculated data shows that the extra growth of the first pillar can be qualitatively described by a geometric model that is based on a $\cos ^{n} \theta$-like emission distribution. The obtained ratio between $\alpha Y$ and $\beta$ is $22 \pm 3$. Calculations in the range of $1<n<2$ yield within $1 \%$ the same curve. Thus, when an ion hits a substrate covered by precursor molecules, the emitted secondary particles can, in principle, deposit 22 times as much material in the proximity of the impact site than at the site itself. Of course, this can only happen when the nearby surfaces are properly oriented and fully covered by the precursor molecules. Likely, redeposition of sputtered atoms and of emitted precursor fragments contributes to this high number. Obviously, also precursor decomposition induced by these impinging particles and by secondary electrons is responsible for the large proximity effect.

Precursor decomposition by heating of the first pillar during the growth of the second one might also explain the broadening. Because heating of the growing pillar itself is small-at most a few degrees-heating by conduction or radiation over a distance of about $1 \mu \mathrm{m}$ or more should have no observable effect. Also the primary beam can induce additional growth of the first pillar. However, ions in the tail of the primary beam are expected to cause an increase in the first pillar height, not its diameter. Scattering of primary ions is mostly forward and therefore one would expect mainly broadening of the pillar base, not the entire pillar. Since alternative mechanisms are improbable, we conclude that the proximity effect is largely caused by secondary particles.

Bending of our IBID pillars is not as large as that of EBID pillars. ${ }^{15,16}$ This difference is not surprising because both Young's modulus and the width of IBID pillars are much higher than those of EBID pillars. ${ }^{24,25}$ The cause of the bending is still under debate. Unlike Kislov and Khodos ${ }^{15}$ and Mitsuishi et al., ${ }^{16}$ we did not observe a clear relationship between pillar bending and substrate conductivity. However, both in our work and in Refs. 15 and 16, pillar broadening is always accompanied by bending. An explanation of the 
bending is the deformation caused by the uneven stress in the first pillar surface due to the additional deposition at one side of the pillar. Considering its unaffected width, it is unlikely that the increased height of the second pillar on $\mathrm{Si}$ or $\mathrm{Cu}$ coated $\mathrm{Si}$ is caused by scattered particles. There must be a different mechanism.

\section{B. Effect of precursor coverage}

The deposition rate of IBID depends strongly on the precursor gas coverage of the substrate surface. As observed, the deposition rate is high when a scanning ion $^{26}$ or electron $^{27}$ beam is moved toward the precursor gas flux. Obviously, the precursor coverage depends on the orientation of the surface with respect to the precursor gas flow. If the surface of a growing structure is not fully exposed to the precursor flux, shadowing occurs; the deposited volume under shadowing is smaller. For high-aspect ratio deposits, such as pillars, shadowing is almost inevitably [see in Fig. 1(a)]. It is important to note that the proximity effect still exists when shadowing is absent [see Figs. 1(b) and 2(a)]. This proximity effect will be explained as below.

From the ratio of the diameter of the nozzle and of the pump inlet, we estimate that the local pressure at the outlet of the nozzle is in the range of $10^{-2}-10^{-3}$ mbar. According to the kinetic gas theory, the corresponding mean free path of $\left(\mathrm{CH}_{3}\right)_{3} \mathrm{Pt}\left(\mathrm{CpCH}_{3}\right)$ precursor molecules at room temperature is roughly $1-10 \mathrm{~mm} .{ }^{18}$ The deposition site was $430 \mu \mathrm{m}$ from the outlet. Therefore, most molecules do not encounter a gas phase collision before impinging onto the surface. Some molecules impinging onto the existing first pillar are reflected back. Others are adsorbed first and then desorbed. These re-entering molecules contribute to the local pressure of the precursor gas near the growing pillar. Assuming uniform reflection and desorption intensities and neglecting dependencies on the polar angle $\theta$, the fraction of molecules that leave the fist pillar and reach the growing one is approximately $D / 2 \pi L$. The extra flux of these molecules can enhance the precursor coverage on the growing pillar, thus enhance the growth speed. The curve in Fig. 2(b) (bottom) is a fit of $\gamma D / 2 \pi L$, with $\gamma$ as a fitting parameter. Agreement is found for $\gamma=1.45$. Because this value is relatively largeeven larger than unity-we conclude that reflection or desorption of precursor molecules constitutes a secondary flux that can have a substantial influence on the growth rate of the growing pillar.

Surface diffusion of absorbed molecules is limited $(<100 \mathrm{~nm}) .^{27}$ Therefore, we conclude that molecules diffusing between the pillars across the substrate surface play a minor role in the precursor coverage enhancement of the growing second pillar. Nevertheless, an accurate estimation of the contributions of all these effects-molecule direct impingement and reflection, absorption, desorption, and diffusion-requires further study. ${ }^{27}$

\section{Effect of charging}

The similarity between pillars grown on bare $\mathrm{Si}$ and on $\mathrm{Cu}$ coated $\mathrm{Si}$ indicates that the conductivity of $\mathrm{Si}$ is sufficient to carry away the surface charges during IBID. However, the charging situation on $\mathrm{Si}_{3} \mathrm{~N}_{4}$ is very different. During IBID pillar growth, the growing pillar is positively charged due to the impingement of positive ions and the emission of secondary electrons. ${ }^{14}$ The insulating substrate, however, captures electrons that are emitted from the pillar or released in the inevitable ion-gas phase collisions. Therefore, the substrate is negatively charged with respect to the pillar. The unequal charge distribution on the equipotential surface of the conductive pillars affects the secondary electron emission. ${ }^{14}$ Secondary electrons are preferably emitted from the central area of the top of the pillar, where the positive charge density and, thus, the surface energy barrier are lowest. Because fewer electrons are emitted from the sidewall, the pillars on an insulating substrate grow faster in height and slower in width. ${ }^{14}$ Moreover, the base of a pillar grown under charging condition has a complex shape, which is possibly caused by a temporary FIB drift during the initial charge buildup. ${ }^{14}$ Since the growing pillar on $\mathrm{Si}_{3} \mathrm{~N}_{4}$ is positively charged, some emitted secondary electrons are attracted back to the pillar and less arrive at the nearby first pillar. This sensitivity on charging suggests that the secondary electrons play an important role in the additional growth nearby. It explains why the broadening of the first pillar is much less on $\mathrm{Si}_{3} \mathrm{~N}_{4}$ than on $\mathrm{Si}$. Moreover, the charging effect can also explain the twisted shape of neighboring pillars on $\mathrm{Si}_{3} \mathrm{~N}_{4}$. At the beginning of growth, both pillars have the same charge and repulsion force bends them apart. In the upper half of the pillar, the bending direction is reversed. Likely, the fixed ion beam shifted the deposition on the top of the bent pillar to the side closest to the first pillar.

The clearly different base shapes, heights, and surface roughness of two neighboring pillars indicate that the surface charging was reduced due to the presence of the first pillar [see Fig. 3(b)]. We conclude that the second pillar becomes shorter and rougher because there was less charging [see Fig. 3(a)]. The effect of charging on IBID pillar growth can also be seen in Fig. 4(c); not only another pillar but also a nearby Pt line can reduce charging during growth considerably.

The reduced charging suggests that transport of electrons between two neighboring pillars takes place. The chance that electrons flow through the insulating $\mathrm{Si}_{3} \mathrm{~N}_{4}$ membrane is very small. However, they can travel through the vacuum from the first pillar to the growing one. We note that diamondlike carbon pillars fabricated by IBID can work as field emitters with a threshold electrical field of about $1 \mathrm{kV} / \mu \mathrm{m} .{ }^{12,13}$ Alternatively, for insulators with secondary electron emission coefficients greater than unity, a succession of electron collisions can effectively result in electrons "skipping" across the surface. ${ }^{28}$

\section{CONCLUSIONS}

We have observed clear proximity effects in IBID of nanopillars grown on a semiconducting bulk $\mathrm{Si}$ and an insu- 
lating $\mathrm{Si}_{3} \mathrm{~N}_{4}$ membrane. When two pillars are grown next to each other, the diameter, height, and shape of the first grown pillar are affected as well as these properties of the second pillar. We conclude that the changes in the first grown pillar are caused by the additional deposition induced by the scattering of secondary particles from the growing second pillar. Deposition by secondary particles in the proximity of the ion-beam impact site can greatly exceed the deposition at the site itself. The changes in the second pillar on Si result from the enhancement of its precursor gas coverage, while on $\mathrm{Si}_{3} \mathrm{~N}_{4}$ they result from variations in substrate charging.

These severe proximity effects diminish the capability of IBID to prototyping structures in the submicrometer range. In order to minimize or prevent detrimental effects, one should make proper choices of conditions and procedures.

\section{ACKNOWLEDGMENTS}

The authors would like to thank V. Kutchoukov (Delft University of Technology in the Netherlands) for $\mathrm{Si}_{3} \mathrm{~N}_{4}$ sample preparation. The authors also thank E. van der Drift, V. Sidorkin, and K. Hagen (all from Delft University of Technology) for their helpful discussions. The authors acknowledge the financial support from NanoNed.

${ }^{1}$ K. Gamo, N. Takakura, N. Samoto, R. Shimizu, and S. Namba, Jpn. J. Appl. Phys., Part 2 23, L293 (1984).

${ }^{2}$ A. Wagner, J. L. Mauer, P. G. Blauner, S. J. Kirch, and P. Longo, J. Vac. Sci. Technol. B 8, 1557 (1990).

${ }^{3}$ S. Matsui, T. Kaito, J. Fujita, M. Komuro, and K. Kanda, J. Vac. Sci. Technol. B 18, 3181 (2000).

${ }^{4}$ T. Morita, R. Kometani, K. Watanabe, and K. Kanda, J. Vac. Sci. Technol. B 21, 2737 (2003)

${ }^{5}$ J. Igaki, K. Nakamatsu, R. Kometani, K. Kanda, Y. Haruyama, T. Kaito, and S. Matsui, J. Vac. Sci. Technol. B 24, 2911 (2006).

${ }^{6}$ J. Igaki et al., Microelectron. Eng. 83, 1221 (2006).

${ }^{7}$ R. Kometani et al., J. Vac. Sci. Technol. B 23, 298 (2005).

${ }^{8}$ R. Kometani et al., Jpn. J. Appl. Phys., Part 1 42, 4107 (2003)

${ }^{9}$ R. Kometani et al., Nucl. Instrum. Methods Phys. Res. B 232, 362 (2005).

${ }^{10}$ R. Kometani et al., Microelectron. Eng. 83, 1642 (2006).

${ }^{11}$ R. Kometani, H. Koike, K. Kanda, Y. Haruyama, T. Kaito, and S. Matsui, Jpn. J. Appl. Phys., Part 1 46, 7963 (2007).

${ }^{12}$ R. Kometani, K. Kanda, Y. Haruyama, T. Kaito, and S. Matsui, Jpn. J. Appl. Phys., Part 2 45, L711 (2006).

${ }^{13}$ R. Kometani, Y. Haruyama, K. Kanda, T. Kaito, and S. Matsui, Jpn. J. Appl. Phys., Part 1 46, 7987 (2007).

${ }^{14}$ P. Chen, H. W. M. Salemink, and P. F. A. Alkemade, Jpn. J. Appl. Phys. 47, 8120 (2008).

${ }^{15}$ N. A. Kislov and I. I. Khodos, Microsc. Microanal. Microstruct. 3, 323 (1992).

${ }^{16}$ K. Mitsuishi, M. Shimojo, M. Takeguchi, M. Tanaka, and K. Furuya, Jpn. J. Appl. Phys., Part 1 45, 5517 (2006).

${ }^{17}$ Y. M. Lau, P. C. Chee, J. T. Thong, and V. Ng, J. Vac. Sci. Technol. B 20 , 1295 (2002).

${ }^{18}$ I. Utke, P. Hoffmann, and J. Melngailis, J. Vac. Sci. Technol. B 26, 1197 (2008).

${ }^{19}$ G. M. Shedd, H. Lezec, A. D. Dubner, and J. Melngailis, Appl. Phys. Lett. 49, 1584 (1986).

${ }^{20}$ P. Chen, P. F. A. Alkemade, and H. W. M. Salemink, Jpn. J. Appl. Phys. 47, 5123 (2008).

${ }^{21}$ J. L. H. Jonker, Philips Res. Rep. 6, 372 (1951).

${ }^{22}$ H. J. Klein, Z. Phys. 188, 78 (1965).

${ }^{23} \mathrm{H}$. Gnaser, in Sputtering by Particle Bombardment, edited by R. Behrisch and W. Eckstein (Springer, Berlin, 2007), p. 275.

${ }^{24}$ S. Okada et al., Jpn. J. Appl. Phys., Part 1 44, 5646 (2005).

${ }^{25}$ S. Okada, T. Mukawa, R. Kobayashi, M. Ishida, Y. Ochiai, T. Kaito, S. Matsui, and J. Fujita, Jpn. J. Appl. Phys., Part 1 45, 5556 (2006).

${ }^{26}$ A. J. DeMarco and J. Melngailis, J. Vac. Sci. Technol. B 17, 3154 (1999).

${ }^{27}$ T. Bret, I. Utke, and P. Hoffmann, Microelectron. Eng. 78-79, 307 (2005).

${ }^{28}$ D. J. Gibbon, in Handbook of Vacuum Physics, edited by A. H. Beck (Pergamon, New York, 1966), Vol. 2, p. 349. 